\title{
COMPACT HOMOGENEOUS ALMOST COMPLEX SPACES OF POSITIVE CHARACTERISTIC $\left({ }^{1}\right)$
}

\author{
BY \\ ROBERT HERMANN(2)
}

1. Introduction. H. C. Wang has classified the compact simply connected homogeneous spaces of positive Euler-Poincaré characteristic admitting a homogeneous complex analytic structure [12]: they are quotients of the semi-simple compact connected Lie groups by subgroups which are centralizers of toral subgroups, and are even homeomorphic to algebraic varieties [8]. We investigate manifolds of this class admitting a homogeneous almost complex structure and find that this classification is in general true, with some of the homogeneous spaces of the compact simple exceptional Lie groups appearing as exceptions. For example, the six sphere is the only sphere of dimension greater than two admitting a homogeneous almost complex structure, as well as the only sphere admitting an exceptional group as a transitive group of motions [1].

Unfortunately, we must define a homogeneous almost complex structure on a manifold as one admitting a transitive Lie group of automorphisms, since it is not known if the group of automorphisms of an almost complex manifold, even if compact, must be a Lie group. The main result is then:

Theorem. Let $G$ be a compact connected Lie group, $L$ a connected closed subgroup containing a maximal torus of $G$ and such that the coset space $G / L$ admits an almost complex structure invariant under $G . G / L$ is then isomorphic to a product of quotients of simple compact connected Lie groups which admit invariant almost complex structures. If then $G$ is simple and $L$ is semi-simple, $G / L$ must be one of nine spaces, with $G$ an exceptional group, whose structure is given more explicitly below. If $G$ is simple and $L$ is not semisimple, $L$ is the centralizer in $G$ of a toral subgroup of $G$ in all but three cases.

While H. C. Wang developed algebraic methods to deal with his problem, here the question is reduced to known facts. In particular we lean heavily on the determination of the maximal subgroups of maximal rank of the compact simple Lie groups done by A. Borel and J. de Siebenthal [3].

I should like to thank here a few people who have greatly helped me in the working out of this paper: D. C. Spencer, for long and tireless help as my

Received by the editors December 9, 1955.

(1) Part of the results of this paper was submitted in a thesis to Princeton University in partial fulfillment of the requirements for the degree of Doctor of Philosophy.

(2) National Science Foundation pre-doctoral fellow, 1953-1955, post-doctoral fellow, 1955-56. 
advisor, C. Ehresmann, J. C. Moore, and A. Borel. I should also like to thank the referee for helpful suggestions.

2. Notations and differential-geometric preliminaries. Let $V$ be a differentiable manifold. (The differentiability class of all manifolds, groups of homeomorphisms, tensor fields, etc. will be understood to be $C^{\infty}$.) If $x \in V$, let $T(x)$ denote the tangent space to $V$ at $x$, defined as in [5, Chap. 3]. Let $T_{p}^{q}(x)$ be the vector space of $p$-covariant, $q$-contravariant tensors on $T(x)$. Let $T_{p}^{q}(V)=\bigcup_{x \in V} T_{p}^{q}(x) . T_{p}^{q}(V)$ can be given a topology in such a way as to be a $C$ differentiable fiber bundle with structural group $G L(n, R)$ and base $V$, the bundle of $(p, q)$ tensors over $V[11] . T(V)=T_{0}^{q}(V)$ is of course called the tangent bundle of $V$. Cross-sections of the bundle $T_{p}^{1}(V)$ are called tensor fields of type $(p, q)$. Let $A_{p}(V)$ be the sub-bundle of $T_{p}^{0}(V)$ formed of all skew-symmetric tensors. The cross-sections of $A_{p}(V)$ are $p$-differential forms on $V$, denoted by $\Gamma\left(A_{p}(V)\right)$, with $\Gamma\left(A_{0}(V)\right)$ interpreted as the set of $C^{\infty}$ real valued functions on $V$. The properties of the Poisson "bracket" operation [ , ]: $\Gamma(T(V)) \otimes_{R} \Gamma(T(V)) \rightarrow \Gamma(T(V))$ and exterior differentiation $d: \Gamma\left(A_{p}(V)\right)$ $\rightarrow \Gamma\left(A_{p+1}(V)\right)$ are well known. The elements of $T_{1}^{1}(x)$ can be interpreted as endomorphisms of $T(x)$ and an $h \in \Gamma\left(T_{1}^{1}(V)\right)$ therefore defines at each point $x \in V$ an endomorphism $h_{x}$ of $T(x)$.

The complex tangent space at $x, C T(x)$, is constructed by extending the field of scalars from $R$ to $C$, i.e. $C T(x)=T(x) \otimes_{R} C$. The corresponding bundles of complex tensors are denoted by $C T(V), C T_{p}^{q}(V)$ and $C A_{p}(V)$. The operation of complex conjugation $-: C \rightarrow C$, extends to $C T_{p}^{q}(x)$, and $T_{p}^{q}(x)$ can be identified with the real elements of $C T_{p}^{q}(x)$, i.e. those invariant by conjugation. The operations [ , ] and $d$ also extend to real operations, i.e. map real tensor fields into real tensor fields.

Let $G$ be a connected Lie group. (\$), the Lie algebra of $G$, will be defined as the $R$-vector space of left invariant vector fields on $G$. Ad $G$ will denote the adjoint representation of $G$ in $\&$ [5]. Let $V$ be a manifold on which $G$ operates as a transitive group of homeomorphisms. If $x \in V$, let $L$ be the subgroup of $G$ leaving $x$ fixed, called the isotropy group at $x$. $V$ can be identified with the coset space $G / L . L$ is said to be reductive in $G$ if there is a subspace $\mathfrak{M}$ of $B$ such that $\mathfrak{B}=\mathfrak{R} \oplus \mathfrak{M}$ and $\operatorname{Ad} L(\mathfrak{M}) \subset \mathfrak{M}$. We will outline the method of reducing the differential geometric structure of $G / L$ to algebraic properties of the Lie algebra of $G$ given essentially by K. Nomizu [9, p. 41]: Since Ad $L$ leaves $\mathfrak{R}$ invariant, there is induced a representation of $L$ into the vector space $(\$) / R$, to be denoted by $\operatorname{Ad}_{G / L} L$. Let $x$ be a point of the coset space $G / L . L$ induces a group of linear transformations on $T(x)$, called the linear isotropy group at $x$, and there is a natural isomorphism between $T(x)$ and $\$ / \&$ such that $L$ acting on $T(x)$ corresponds to $\operatorname{Ad}_{G / L} L$. A tensor field on $G / L$ invariant by $G$ gives rise to a tensor on $\mathbb{S} / \mathbb{R}$ invariant by $\operatorname{Ad}_{G / L} L$, and conversely.

All that we have said in the last paragraph has its obvious analogue for the 
complex tangent space, e.g. $C T(x)$ is isomorphic to $C \otimes G / L=C(S / C R$, etc.

3. Almost complex structures. We follow C. Ehresmann's definition [6] of an almost complex structure alternately in real or complex terms.

DEFINITION 3.1. An almost complex structure on a manifold $V$ is defined by an $h \in \Gamma\left(T_{1}(V)\right)$ such that $h_{x} \circ h_{x}=-$ (identity) for each $x \in V$.

DEFINITION 3.1'. An almost complex structure on a manifold $V$ is defined by a splitting of $C T(V)$ as the Whitney sum of two complex vector bundles $T^{0,1}(V)$ $\oplus T^{1,0}(V)$ such that

$$
T^{1,0}(x)=T^{0,1} x
$$

for each $x \in V$.

This decomposition of $C T(V)$ defines a decomposition of $C A_{p}$ into "types," $C A_{p}(V)=\sum_{r+s=p} A_{r, s}(V)$, such that $\bar{A}_{r, s}(V)=A_{s, r}(V)$, familiar in the theory of Kahler manifolds [13].

Recall that a Riemannian metric on $V$ is defined by a $q \in \Gamma\left(T_{2}^{0}(V)\right)$ such that the induced bilinear form $q_{x}$ on $T(x)$ is positive definite and symmetric for each $x \in V$. If $h$ defines an almost complex structure on $V$, an Hermitian metric is defined by a Riemannian metric $q$ satisfying the additional requirement: $q_{x}\left(t, h_{x}(t)\right)=0$ for all $t \in T(x), x \in V$.

The two-form $w_{x}$ defined by $(s, t) \rightarrow q_{x}\left(s, h_{x}(t)\right)$ for each $s, t \in T(x)$ is then skew-symmetric and defines a two-differential form on $V$, called the fundamental form of the Hermitian metric. The Hermitian metric is said to define a Kahler metric if the fundamental form $w$ is closed, i.e. $d w=0$. In this case, $w^{k}$, for $1<k<$ complex dim. of $V$, cannot be a derived form if $V$ is compact [13].

An almost complex structure on a manifold $V$ is said to be homogeneous if there is a transitive Lie group $G$ of automorphisms. $G$ is said to leave the almost complex structure invariant. From the discussion above, we see that an invariant almost complex structure on a homogeneous space $G / L, G$ a connected Lie group, is defined by an endomorphism $h:(B) / \mathbb{R} \rightarrow \mathbb{B} / \mathbb{R}$ such that (1) $\operatorname{Ad}_{G / L} L$ commutes with $h$ and (2) $h^{2}=-$ (identity), or, alternatively, by a decomposition $C S / C R=\mathfrak{M}_{1,0} \oplus \mathfrak{M}_{0,1}$ such that (1) $\overline{\mathfrak{M}}_{0,1}=\mathfrak{M}_{1,0}$ and (2) $\operatorname{Ad}_{G / L} L\left(\mathfrak{M}_{0,1}\right) \subset \mathfrak{M}_{0,1}$.

Given a manifold $V$, A. Borel and J.-P. Serre have proven that a field of endomorphisms $x \rightarrow h_{x}$ of $T(V)$ such that $h_{x}$ has no real eigenvalues defines an almost complex structure on $V$ in a canonical way $[2$, p. 436]. Similarly, one proves the following

Lemma 3.1. Let $G$ be a connected Lie group, $L$ be a closed subgroup. An endomorphism $h: \$ / R \rightarrow\left(S / R\right.$ such that (1) $h$ commutes with $\operatorname{Ad}_{G / L} L$ and (2) $h$ has no real eigenvalues, defines in a canonical way an invariant almost complex structure on $G / L$.

LEMMA 3.2. Let $G$ be a connected Lie group, $L$ be the centralizer in $G$ of an 
element $g \in G$ of odd order or of a toral subgroup $T$ of $G$. Then $G / L$ admits an invariant almost complex structure.

Proof. In the first case, $\operatorname{Ad}_{G / L} g$ has no real eigenvalues and commutes with $\operatorname{Ad}_{G / L} L$, by the definition of $L$. In the second case, choose an element $g \in T$ such that the powers of $g^{2}$ are everywhere dense in $T$. Again $\operatorname{Ad}_{G / L} g$ has no real eigenvalues and so leads to an invariant almost complex structure on $G / L$, by Lemma 3.1 .

We now briefly discuss the idea of an almost complex analytic function on an almost complex manifold, generalizing the classical idea of complex analytic function [14].

Definition 3.2. Let $V$ be an almost complex manifold, $U$ be an open set of $V$. A complex-valued $C^{\infty}$ function $f$ on $U$ is said to be an almost complex analytic function if $d f \in A_{1,0}(y)$ at each $y \in U$.

The necessary and sufficient condition that a given almost complex structure on a manifold $V$ of (real) dimension $2 n$ be derivable from a complex analytic structure on $V$ is that, for each $x \in V$, there exist $n$ almost complex analytic functions $f_{1}, \cdots, f_{n}$ defined in a neighborhood of $x$ such that the co-vectors $d f_{1}, \cdots, d f_{n}$ at $x$ span $A_{1,0}(x)$. The functions $f_{1}, \cdots, f_{n}$ are then taken as a base for the complex analytic functions about $x$, to define a complex manifold in the classical sense [14].

At the other extreme to the complex analytic manifolds stand those almost complex manifolds which admit locally no almost complex functions. For example, C. Ehresmann has proved that the six-sphere, with the almost complex structure derived from the Cayley numbers, admits no functions (unpublished).

THEOREM 3.1. Let $G$ be a connected Lie group, $L$ a closed subgroup such that the linear isotropy group of $G / L$ is irreducible and suppose that $G / L$ admits an invariant almost complex structure. Then, either there are locally no almost complex analytic functions or the almost complex structure derives from a complex analytic structure.

Proof. Suppose the dimension of $G / L$ is $2 n$. Given $x \in G / L$, it suffices to prove that there are either no nonconstant almost complex functions defined locally about $x$ or $n$ functionally independent ones, since functions about $x$ can be transported by operations of $G$ to any other point of $G / L$. If $f$ is such a function, and $f_{g}$ denotes the transform of $f$ by the transformation induced by $g \in L$, note that the subspace of $A_{1,0}(x)$ spanned by all covectors of the form $d f_{o}$, for all $g \in L$, is invariant by the linear isotropy group at $x$ (if $L$ is identified with the isotropy group at $x$ ), hence must equal $A_{1,0}(x)$, implying that there are $n$ functionally independent almost complex analytic functions defined about $x$.

REMARK. To see how this applies to the six-sphere, it is well known that the compact simple Lie group $G_{2}$ acts on $S_{6}$, with isotropy group $S U(3)$. Since 
$S_{6}$ can admit no field of $k$-planes, $0<k<6[11$, p. 144$]$, one sees that the linear isotropy group is irreducible. The center of $S U(3)$ is $Z_{3}$, the cyclic group of order 3, and it follows from the results of Borel and de Siebenthal [3] that $S U(3)$ is the centralizer of its own center in $G_{2}$, and then, by Lemma 3.2, $G_{2} / S U(3)$ admits an invariant almost complex structure. This structure cannot derive from a complex analytic structure, by the results of $\mathrm{H}$. C. Wang [12], hence by Theorem 3.1, there are no almost complex analytic functions.

This group-theoretical proof of the existence of an almost complex structure on $S_{6}$ will later be generalized to apply to certain of the other quotients of the compact simple exceptional Lie groups by nonsymmetric maximal subgroups of maximal rank. In all, there are six such spaces that share with $S_{6}$ the property of having irreducible linear isotropy groups.

\section{Symmetric subgroups.}

Definition 4.1. Let $G$ be a connected Lie group, $L$ be a closed subgroup. $L$ is said to be a symmetric subgroup of $G$ if there is an automorphism $S$ of $(B)$ such that

(1) $\mathbb{R}$ is the set of elements of $(5)$ left fixed by $S$, and

(2) $S^{2}=$ identity.

In this case the homogeneous space $G / L$ is said to be symmetric $[4 ; 9]$.

We say that a subgroup $L$ of a Lie group $G$ is maximal in $G$ if $\mathbb{R}$ is a maximal subalgebra of (5. E. Cartan has proved $[4$, p. 52] that, if $G$ is a connected Lie group and $L$ is a compact symmetric subgroup, the linear isotropy group of $G / L$ is irreducible, and hence in particular $L$ is a maximal subgroup of $G$.

The following result is due to A. Borel.

Proposition 4.1. Let $G$ be a connected Lie group, $L$ be a compact symmetric subgroup. If $G / L$ admits an invariant almost complex structure, it admits an invariant Kahler metric.

Proof. If $h:(S) / \& \rightarrow(\mathfrak{S} / \mathbb{R}$ defines an invariant almost complex structure on $G / L$, we construct a positive definite quadratic form $q$ on $(S / \&$ such that (1) $q(t, h(t))=0$ for $t \in \mathbb{S} / \mathcal{R}$ and (2) $q$ is invariant by $\operatorname{Ad}_{G / L} L$. It is clear that a form $q$ exists satisfying (1). The usual trick of integrating this form over $L$ using the Haar measure on $L$ gives a form satisf ying (1) and (2).

This form defines an invariant Hermitian metric on $G / L$. The fundamental two-form of this Hermitian metric is invariant by $G$, hence its exterior derivative is zero $[9$, p. 54$]$, hence the metric is a Kahler metric.

Proposition 4.2. Let $G$ be a connected compact simple Lie group, $L$ be a symmetric subgroup. Then $G / L$ admits an invariant almost complex structure if and only if $L$ is not semi-simple.

Proof. The necessity is clear, since the second Betti number of $G / L$ is zero if and only if $L$ is semi-simple. Conversely, suppose that $L$ is not semisimple. As we have remarked, $L$ is maximal in $G$. Since center $L$ contains a 
toral subgroup, $L$ is the centralizer of a toral subgroup and Lemma 3.2 applies.

5. Homogeneous almost complex structures on compact simply connected spaces of positive Euler-Poincare characteristic. Recall that the quotient of a compact connected Lie group $G$ by a closed subgroup $L$ is of positive EulerPoincaré characteristic if and only if $L$ contains a maximal torus of $G$. All maximal toral subgroups of $G$ are conjugate. The following lemma is well known, and reduces the study of compact simply connected homogeneous spaces of positive characteristic to the product of irreducible spaces, i.e. quotients of the compact simple Lie groups by subgroups of maximal rank.

LemMA 5.1. If $G$ is a compact connected Lie group and is the direct product of invariant subgroups $G^{\prime}$ and $G^{\prime \prime}$, and $L$ is a subgroup of $G$ containing a maximal $t$ orus of $G$, then $L$ is the direct product of $G^{\prime} \cap L$ and $G^{\prime \prime} \cap L[3$, p. 210].

We summarize some of the facts about the structure of subgroups of maximal rank of the compact connected simple groups proved by $\mathrm{A}$. Borel and J. de Siebenthal [3] that we will need.

5.2. Let $G$ be a compact connected simple Lie group, L a connected subgroup of maximal rank, i.e. $L$ contains a maximal torus of $G . L$ is then centralizer in $G$ of its own center. The maximal subgroups of maximal rank are centralizers in $G$ of elements of finite prime order, and indeed, if $G$ is a classical group, of elements of order two. There are only a finite number of conjugacy classes of subgroups of maximal rank of $G$. If $G$ is an exceptional group, a maximal subgroup of maximal rank is symmetric if its Lie algebra is isomorphic to that of a symmetric subgroup of $G$. The structures possible for $B / R$ if $L$ is a maximal nonsymmetric subgroup of maximal rank are: $G_{2} / A_{2}, F_{4} /\left(A_{2}\right)^{2}, E_{6} /\left(A_{2}\right)^{3}, E_{7} / A_{2}$ $\times A_{5}, E_{8} / A_{8}$ or $A_{2} \times E_{6}$ or $A_{4} \times A_{4}$.

The last two statements are not quite proved in [3], but one has simply to verify in each case listed in [3, p. 220] that is isomorphic to a symmetric subgroup that there is an element of order two in its center.

The following theorem shows that the question of the existence of an invariant almost complex structure on a homogeneous space $G / L, G$ compact connected, $L$ a subgroup of maximal rank can be reduced to the case where $G$ is simple and $L$ a maximal subgroup.

Proposition 5.3. Let $G$ be a compact connected Lie group, $L$ be a connected subgroup containing a maximal torus of $G$, contained in a chain of subgroups $L \subset L^{\prime} \subset \cdots \subset L^{m} \subset G$, with $L$ a maximal subgroup of $L^{\prime}, \cdots, L^{m}$ a maximal subgroup of $G$. If $G / L$ admits an invariant almost complex structure, so does $L^{\prime} / L$.

The proof is by induction on $m$. Let $T$ be a maximal torus of $G$ contained in $L$. Choose an element $g \in T$ such that $L^{m}$ is the centralizer of $g$ in $G$, in 
accordance with 5.2. Choose a subspace $\mathfrak{M}$ of $\&$ such that $\mathbb{S}=\mathfrak{R}^{m} \oplus M$, Ad $L^{m}(\mathfrak{M}) \subset \mathfrak{M}$, and a subspace $\mathfrak{R}$ of $\mathfrak{R}^{m}$ such that $\mathfrak{R}^{m}=\mathfrak{R} \oplus \mathfrak{N}$, Ad $L(\mathfrak{R}) \subset \mathfrak{N}$. An almost complex structure on $G / L$ is defined by an endomorphism $h$ of $\mathfrak{N} \oplus \mathfrak{M}$ such that (1) $h^{2}=-$ (identity) and (2) Ad $L \circ h=h \circ \operatorname{Ad} L$.

We assert that $h$ leaves $\mathfrak{N}$ invariant, and so induces an almost complex structure on $L^{m} / L$, to complete the proof by induction. For, if $\mathfrak{n} \in \mathfrak{N}$, $\operatorname{Ad} g(\mathfrak{n})$ $=\mathfrak{n}$. Since $g \in L, \operatorname{Ad} g \circ h=h \circ \operatorname{Ad} g$, and $h(\mathfrak{n})$ is left fixed by Ad $g$ and so must belong to $\mathfrak{R}$.

CoROllary 5.4. If $G$ is a direct product of $G^{\prime}$ and $G^{\prime \prime}$, any invariant almost complex structure on $G / L$ is isomorphic to the product of invariant almost complex structures on $G^{\prime} / G^{\prime} \cap L$ and $G^{\prime \prime} / G^{\prime \prime} \cap L$.

The question of the existence of an invariant almost complex structure if $L$ is a maximal subgroup can be settled immediately.

THEOREM 5.1. Let $G$ be a compact connected simple group, $L$ be a maximal connected semi-simple subgroup of maximal rank. $G / L$ admits an invariant almost complex structure if and only if $L$ is not a symmetric subgroup of $G$.

Proof. Suppose $L$ is not a symmetric subgroup of $G$. Center $L /$ center $G$ cannot contain an element of even order, hence by 5.2 must contain an element of odd order. By Lemma 3.2, $G / L$ must admit an invariant almost complex structure. The converse now follows from Proposition 4.2.

We can now state as a consequence of what has been proved the generalization of Wang's theorem that a compact simply connected space of positive characteristic and zero second Betti number can admit no homogeneous complex analytic structure.

TheOREM 5.2. Let $G$ be a connected compact Lie group, $L$ be a connected semi-simple subgroup of maximal rank such that $L$ is contained as a symmetric subgroup in a connected subgroup of $G$. Then, $G / L$ admits no invariant almost complex structure. This condition is always verified if $G$ is a classical group.

We must now deal with the cases that escape Theorem 5.2.

Lemma 5.5. Let $G$ be a compact connected simple Lie group, $L$ be a connected subgroup of maximal rank such that $L$ contains an exceptional Lie algebra as ideal. Then, $L$ is a maximal subgroup of $G$, with an exception of $B=E_{8}$, $\mathfrak{R}=A_{1} \times E_{6} \times T_{1}$ or $E_{6} \times T_{2}$.

Proof. We proceed by induction on the dimension of $G / L$. If $L$ is not maximal in $G, L \subset M \subset G$, with $M$ a subgroup of maximal rank of $G$ such that $L$ is maximal in $M$. Since from the table [3, p. 220], the classical groups can contain no subgroups of maximal rank whose Lie algebras contain exceptional ideals, $\mathfrak{M}$ must contain an exceptional algebra as ideal and, by induction hypothesis, we can assume that $M$ is maximal in $G$ or that $M$ has the 
structure $E_{6} \times T_{2}$ or $A_{1} \times E_{6} \times T_{1}$. Since $E_{6}$ has only classical groups as maximal subgroups of maximal rank, $\mathfrak{R}=\mathfrak{M}$ in all cases but $\mathfrak{M}=A_{1} \times E_{7}$. This possibility leads to $\mathbb{R}=A_{1} \times E_{6} \times T_{1}$ or $E_{6} \times T_{2}$, which are our allowed exceptions.

Theorem 5.3. Suppose $G / L$ is a homogeneous space admitting an invariant almost complex structure with $G$ and $L$ compact, connected Lie groups and with $G$ simple. Then, if $L$ is semi-simple, it is a maximal nonsymmetric subgroup of $G$, whose structure is described in 5.2 , with exceptions for $\left(B=E_{8}, \&=A_{1} \times A_{2}\right.$ $\times A_{5}$ or $\left(A_{2}\right)^{4}$. If $\mathbb{R}$ is not semi-simple, then $L$ is the centralizer in $G$ of a toral subgroup of $G$, with the exceptions: $(B)=E_{7}, \mathbb{R}=\left(A_{2}\right)^{3} \times T_{1}, \quad B=E_{8}, \quad \mathbb{R}=A_{1} \times\left(A_{2}\right)^{3}$ $\times T_{1}$, or $\mathbb{R}=\left(A_{2}\right)^{3} \times T_{2}$. Further, there are homogeneous spaces having these structures and admitting invariant almost complex structures.

Proof. First, suppose $L$ semi-simple. If $L$ is not maximal in $G, L \subset M \subset G$, with $L$ maximal in $M . M$ must be semi-simple and $M / L$ admits an invariant almost complex structure by Proposition 5.3. $\mathfrak{M}$ contains then an exceptional Lie algebra as ideal, and $M$ is maximal in $G$, by Lemma 5.5 . The only possibilities of structure for $\mathfrak{B}$ and $\mathfrak{M}$ are now: $\left(\mathfrak{S}=E_{8}, \mathfrak{M}=A_{2} \times E_{6}\right.$ or $A_{1} \times E_{7}$ and $\&$ must be then $A_{1} \times A_{2} \times A_{5}$ or $\left(A_{2}\right)^{4}$.

Now suppose $\mathfrak{R}$ non-semi-simple, and let $T$ be the connected component of the identity of the center of $L$. T is then a toral subgroup of $G$. Let $K$ be the centralizer of $T$ in $G$. $K$ is connected. If $L \neq K$, let $M$ be a subgroup of $K$ such that $L$ is maximal in $M . M / L$ admits then an invariant almost complex structure, the connected component of the center of $M$ is $T$. Putting $M^{\prime}$ $=M / T$ and $L^{\prime}=L / T, M / L$ is isomorphic to $M^{\prime} / L^{\prime}$, which admits then an invariant almost complex structure. $\mathfrak{M}^{\prime}$ contains then an exceptional algebra as ideal and Lemma 5.5 applies: $\mathfrak{H}=E_{7}, \mathfrak{M}=E_{6} \times T_{1}, \quad\left(S=E_{8}, \mathfrak{M}=A_{1} \times E_{6}\right.$ $\times T_{1}$ or $E_{6} \times T_{2}$. Determining the structure of $\mathbb{R}^{\prime}$ from the first part of this proof, we see that $\&$ must have struture $\left(A_{2}\right)^{3} \times T_{1}, A_{1} \times\left(A_{2}\right)^{3} \times T_{1}$ or $\left(A_{2}\right)^{3}$ $\times T_{2}$.

To deal with the converse, we make the following remark: If $L \subset M \subset G$ are connected compact Lie groups, and if $G / M$ and $M / L$ admit invariant almost complex structures, so does $G / L$. Using the construction of maximal subgroups of maximal rank of the simple groups given by Borel and de Siebenthal [3], we see in each case that there are groups $L \subset M \subset G$, with $G$ simple, $L$ maximal in $M$ and $M$ maximal in $G$, such that $M / L$ admits an invariant almost complex structure, and $G$ and $L$ have the desired structure. For all but the case $\mathbb{S}=E_{8}, \mathfrak{l}=A_{1} \times A_{2} \times A_{5}, \mathfrak{R}=A_{1} \times E_{7}, G / M$ and hence also $G / L$ has an invariant almost complex structure. Dealing with this case then in more detail, we see from [3, p. 221] that if $G$ is the adjoint group of $E_{8}, M$ has center $Z_{2}$. In the adjoint group of $A_{1} \times E_{7}$, there exists a maximal subgroup $K$ of structure $A_{1} \times A_{2} \times A_{5}$ and with center $Z_{3}$. Let $L$ be the inverse image of $K$ under the covering map $M \rightarrow$ Ad $M$. L has center $Z_{3} \times Z_{2}$, and hence 
there exists a $g \in$ center $L$ having order 3 which does not lie in center $M$. It is easily verified that $L$ is the connected component of the centralizer of $g$ in $G$, and so again $G / L$ admits an invariant almost complex structure by Lemma 3.2 .

6. We now turn as promised to the question of whether the linear isotropy group of the quotients of the compact simple groups by maximal nonsymmetric subgroups of maximal rank is irreducible. First, we must go into the theory of the roots of complex semi-simple Lie algebras. For convenience, we follow the terminology in [10].

Let $G$ be a compact connected simple Lie group, $T$ a maximal toral subgroup. $C \mathfrak{T}$ is a Cartan subalgebra of $C(S$. Let $A$ be the set of roots of $C(S)$ with respect to $C \mathfrak{T}$, a set of linear forms on $C \mathfrak{T}$. Let $R A$ be the real vector space of linear combinations of the roots with real coefficients. Let $n$ be the real

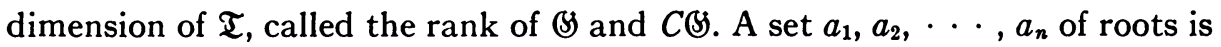
called a simple root system if any root is a linear combination with intregral coefficients all of the same sign of the $a_{1}, \cdots, a_{n}$. The existence of at least one simple root system is well known. A Weyl basis for $C(S)$ is a collection of nonzero elements of $C G, e_{a}$ and $h_{a}$, indexed by the roots $a$, such that (1) $h_{a} \in C \mathfrak{T},(2)\left[h_{a}, e_{b}\right]=\langle a, b\rangle e_{b}$ for all $a, b \in A$, where $\langle$,$\rangle is the quadratic form$ induced on $R A$ by the Killing form of $C(S)$ (known to be positive definite and to take rational values on $A \times A)$ and (3) if $a, b$ and $a+b \in A$, and $\left[e_{a}, e_{b}\right]$ $=N_{a, b} e_{b}$, then $N_{a, b}=N_{-a,-b}$. It is known that there exists at least one Weyl basis such that $B$ is spanned by the elements $e_{a}+e_{-a}, i\left(e_{a}-e_{-a}\right)$ and $i h_{a}$ for $a \in A$. (cf. [10, Exp. II, p. 9]). If $a_{1}, \cdots, a_{n}$ is a simple root system for $C(S)$, and $a \in A$, set $m_{i}(a)$ equal to the $i$ th component of $a$, i.e. $a=\sum_{1 \leqq i \leqq n} m_{i}(a) a_{j}$. If $a$ and $b \in A$, then

$$
-2\langle a, b\rangle /\langle a, a\rangle=c_{a, b}=-p+q,
$$

where $p$ (resp. $q$ ) is the largest integer such that $b-p a$ (resp. $b+q b)$ is a root.

Lemma 6.1. Suppose $a_{1}, \cdots, a_{n}$ is a simple root system of $C(5)$ and suppose that $b$ is a root with $m_{1}(b)<0$ and $\left\langle a_{i}, b\right\rangle \leqq 0$ for $i>1$. Then, $m_{i}(b)<0$ for $i>1$. Note that a minimal negative root satisfies this condition.

Proof. We can suppose that $m_{i}(b)<0$ for $i \leqq k$ and that $m_{i}(b)=0$ for $i>k$, with $1<k<n$. For $i>k, 0 \leqq\left\langle b, a_{i}\right\rangle=\sum_{1 \leqq j \leqq k} \mid m_{j}(b)\left\langle a_{j}, a_{i}\right\rangle \geqq 0$, hence $\left\langle a_{j}, a_{i}\right\rangle=0$ for $i>k, j \leqq k$, contradicting that $C B$ is simple.

TheOREM 6.1. Let $G$ be a compact connected simple Lie group, $L$ be a maximal subgroup which is the connected component of the centralizer in $G$ of an element of order 3. Then, the isotropy group of $G / L$ is irreducible.

Proof. From [3] we know that $C \&$ can be constructed in the following way; there is a simple root system $a_{1}, \cdots, a_{n}$ of $C(5)$ and a root $a_{0}$ minimal with respect to the lexicographic ordering of $A$ determined by $a_{1}, \cdots, a_{n}$ such 
that, if $B$ is the set of roots of $C B$ which are the sum with integral coefficients of $a_{0}, a_{2}, \cdots, a_{n}$, then $C$ ? is spanned by $C \mathfrak{T}$ and $e_{b}$, for $b \in B$. In addition, $m_{1}\left(a_{0}\right)=-3$. Let $D$ be the set of all roots $a$ such that $a \notin B$, i.e. all roots $a$ such that $0<\left|m_{1}(a)\right|<3$. Let $\mathbb{S}=\mathfrak{R} \oplus \mathfrak{M}$ be a reduction of $\mathfrak{R}$ in $(\mathfrak{s}$ i.e. Ad $\mathfrak{R}(\mathfrak{M})$ $\subset \mathfrak{M}$. Clearly $C \mathfrak{M}$ is spanned by all $e_{a}$ for $a \in D$. Let $\mathfrak{M}_{1,0}$ be the subspace of $C \mathfrak{M}$ spanned by all $e_{a}$ with $m_{1}(a)=1$ or -2 and let $\mathfrak{M}_{0,1}$ be the subspace of $C \mathfrak{M}$ spanned by $e_{a}$ with $m_{1}(a)=-1$ or 2 . It is clear that $\overline{\mathfrak{M}}_{0,1}=\mathfrak{M}_{1,0}$ and that Ad $L\left(\mathfrak{M}_{1,0}\right) \subset \mathfrak{M}_{1,0}$ i.e. the subspaces determine an invariant almost complex structure on $G / L$. This almost complex structure is easily seen to be identical with that constructed by means of Lemma 3.2. To prove the theorem then we must prove that $\mathrm{Ad} C \mathbb{R}$ acting in $\mathfrak{M}_{1,0}$ is irreducible.

Now, $e_{a_{1}} \in \mathfrak{M}_{1,0}$. Let $\mathfrak{R}$ be the smallest subspace of $\mathfrak{M}_{1,0}$ containing $e_{a_{1}}$ and invariant by $\operatorname{Ad} C R$. Suppose then that $\mathfrak{N} \neq \mathfrak{M}_{1,0}$. $\mathfrak{N}$ is spanned by root vectors. Let $D^{\prime}$ be the set of all roots $a$ such that $e_{a} \in \Re$. Let $D^{\prime \prime}$ be the set of roots $a$ such that $e_{a} \in \mathfrak{M}_{1,0}$ but $a \in D^{\prime}$. Let $\mathfrak{P}$ be the subspace of $\mathfrak{M}_{1,0}$ spanned by the $e_{a}, a \in D^{\prime \prime}$. Then $\mathfrak{M}_{1,0}=\mathfrak{N} \oplus \mathfrak{P}$ and $\operatorname{Ad} C \mathfrak{R}(\mathfrak{P}) \subset \mathfrak{P}$.

We assert first that $D^{\prime \prime}$ contains no positive roots. For, otherwise let $a$ be a minimal positive root in $D^{\prime \prime}$. It is clear that $a$ is not a simple root. Then $a=a^{\prime}+a^{\prime \prime}$, with $a^{\prime}$ and $a^{\prime \prime}$ positive roots. Then $1=m_{1}(a)=m_{1}\left(a^{\prime}\right)+m_{1}\left(a^{\prime \prime}\right)$. Let us say then that $m_{1}\left(a^{\prime}\right)=1$ and $m_{1}\left(a^{\prime \prime}\right)=0$. Then $a^{\prime \prime} \in B$ and $a^{\prime} \in D^{\prime}$. Then $N_{a^{\prime}, a^{\prime \prime}} e_{a}=\left[e_{a^{\prime}}, e_{a^{\prime \prime}}\right] \in \mathfrak{N}$ and $N_{a^{\prime}, a^{\prime \prime}} \neq 0$ since $a^{\prime}+a^{\prime \prime}$ is a root, contradiction.

$D^{\prime \prime}$ is composed then wholly of negative roots. Let $-a$ be a maximal negative root in $D^{\prime \prime}$. First, $-a \pm a_{0}$ is not a root. $m_{1}(a)=2$ and $a-a_{i}$ is not a root for $i>1$ and hence, setting $q_{i}=-2\left\langle a, a_{i}\right\rangle /\left\langle a_{i}, a_{i}\right\rangle$ for $1 \leqq i \leqq n$,

6.2 .

6.3 . $q_{i} \geqq 0$ for $2 \leqq i \leqq n$, and

$$
\left\langle a, a_{0}\right\rangle=0 \text {. }
$$

Now $a=\sum_{1 \leqq i \leqq n} m_{i}(a) a_{i}$, hence

6.4 .

$$
q_{i}=\sum_{j \neq i} m_{j}(a) c_{a_{i}, a_{j}}-2 m_{i}(a) \text { for } 1 \leqq i \leqq n .
$$

We assert that $a+a_{1}$ is not a root. Otherwise, $m_{1}\left(a+a_{1}\right)=3$, hence $b=-a-a_{1} \in B$ and $-a=b+a_{1}$, i.e. $e_{-a} \in N^{*}$, contradiction. Then, $q_{1}=0$ or -1 . We assert that $q_{1}=-1$. Otherwise, $a_{1}=1 / 3\left(a_{0}-\sum_{i>1} m_{i}\left(a_{0}\right) a_{i}\right)$, hence $0=\left\langle a, a_{1}\right\rangle=\sum_{i>1} m_{i}\left(a_{0}\right)\left\langle a_{i}, a_{1}\right\rangle$. But $m_{i}\left(a_{0}\right)<0$ and $\left\langle a_{i}, a_{1}\right\rangle \leqq 0$ for $i>1$, implying that $\left\langle a_{i}, a_{1}\right\rangle=0$ for $\left.i\right\rangle 1$, contradicting that $C G$ is simple.

To complete the proof of the theorem one must now go through the list of the maximal subgroups which are centralizers of elements of order 3 and verify in each case, of which there are seven in all, that the conditions we have written down that the root $a$ must satisfy are incompatible. This straightforward though long computation will be left out here.

REMARK. It can be shown that the linear isotropy group of the quotient of a simple compact group by a maximal semi-simple subgroup which is the 
centralizer of an element of order 5 is not irreducible. However, this class of spaces has only two members.

\section{BIBLIOGRAPHY}

1. A. Borel, Some remarks about Lie groups transitive on spheres and tori, Bull. Amer. Math. Soc. vol. 55 (1949) pp. 580-587.

2. A. Borel and J.-P. Serre, Groupes de Lie et puissances reduites, Amer. J. Math. vol. 75 (1953) pp. 409-448.

3. A. Borel and J. de Siebenthal, Les sous-groupes fermés de rang maximum des groupes de Lie clos, Comment. Math. Helv. vol. 23 (1949) pp. 200-221.

4. E. Cartan, La therorie des groupes finis et continus et l'analysis situs, Mémorial Sci. Math., Paris, 1930.

5. C. Chevalley, Lie groups, I, Princeton, 1946.

6. C. Ehresmann, Sur les variettes presques complexes, Proceedings of the International Congress of Mathematicians, vol. 2 (1950) pp. 412-419.

7. A. Fröhlicher, Zur Differentialgeometrie der komplexen Strukturens, Math. Ann. vol. 129 (1955) pp. 50-95.

8. M. Goto, On algebraic homogeneous spaces, Amer. J. Math., vol. 76 (1954) pp. 811-818.

9. K. Nomizu, Invariant affine connections on homogeneous spaces, Amer. J. Math. vol. 76 (1954) pp. 33-65.

10. Seminaire "Sophus Lie," Algèbres de Lie et topologie des groupes de Lie, Ann. Ecole Norm. Paris, 1954-1955.

11. N. Steenrod, Topology of fiber bundles, Princeton, 1951.

12. H. C. Wang, Closed manifolds with homogeneous complex structures, Amer. J. Math. vol. 76 (1954) pp. 1-32.

13. A. Weil, Theorie der Kählerschen Mannigfaltigkeiten, Göttingen, 1953.

14. H. Weyl, Die Idee der Riemannschen Fläche, Berlin, 1912.

Princeton University,

Princeton, N.J.

University of Chicago,

Chicago, Ill 